\section{§9. Investigation of Crystal Structure Formation under Microwave Heating}

Takayama, S.,

Kakurai, K., Takeda, M. (JAEA),

Matsubara, A. (Chubu Univ.),

Nishihara, Y. (College of Science, Ibaraki Univ.),

Nishijo, J. (Institute for Molecular Science),

Sano, S. (Advanced Industrial Science and

Technology),

Nishi, N. (Institute for Molecular Science),

Sato, M.

\begin{abstract}
Recently, we have developed new methods to form nano-structures consisting of small magnetic domains of $5 \sim 20$ nanometers with random orientations, when some ferromagnetic materials are exposed to the magnetic field with microwave frequencies. To investigate the consequences of the non-equilibrium reactions by electromagnetic wave energy, we performed polarized neutron diffraction measurements to characterize the magnetic nanodomain structure of the microwave heated magnetite powder by using the polarized neutron triple-axis spectrometer TAS-1 at JRR-3 of Japan Atomic Energy Agency. In the magnetite samples heated under magnetic field of microwave up to $1200{ }^{\circ} \mathrm{C}$, magnetic Bragg peaks arising from crystalline magnetite of at least 20 nanometer in size were observed.
\end{abstract}

Keywords

Magnetic Heating, Nano-domein,

\section{Introduction}

The microwave processes were not applied the powder metallurgy for short microwave skin depth of a bulk metal. R.Roy et al. first reported sintering of metal powders by microwave in 1999, and the decrystallization of ferrite magnetic materials by heating in microwave magnetic field in 2002.

Recently, we have developed new methods to form nano-structures consisting of small magnetic domains of $5 \sim 20$ manometers with random orientations, when some ferromagnetic materials are exposed to the magnetic field at microwave frequencies.To characterize the magnetic nanodomain structures of these microwave magnetic field heat treated samples, polarized neutron diffraction experiment was performed.

\section{Experimental Setup}

Polarized neutron diffraction profiles were measured by using the thermal neutron triple-axis spectrometer TAS- 1 installed at the $2 \mathrm{G}$ beam port of the research reactor JRR-3 at the Japan Atomic Energy Agency (JAERI) in Tokai. To measure the spin dependent magnetic scattering, Bragg reflected neutron intensities from the sample are recorded with the incident neutrons polarized by means of magnetized Heusler alloy monochromator. The spin state of the incident neutrons could be selected by operating a direct current flipper to be either parallel (flipper on) or antiparallel (flipper off) with respect to the guide field. The energy of the inicident neutrons was $14.7 \mathrm{meV}$ and pyrolytic graphite filter was inserted to remove the higher order contaminations. The sample was at RT and fully magnetized under 1T magnetic field applied vertically to the scattering plane by an electromagnet on the sample table.

\section{Experimental Results}

Figures 3 show the neutron scattering profiles of the magnetite (220) Bragg reflections of the $1200{ }^{\circ} \mathrm{C}$ microwave heated samples. These resolution limited polarization dependent Bragg reflections unambiguously indicate the existence of the ferromagnetic long range order in magnetite with at least $20 \mathrm{~nm}$ correlation length.

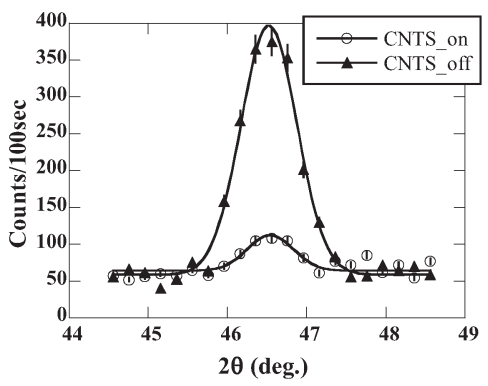

Figure 1: Polarized Neutron Scattering profile of samples heated by magnetic field at $1200^{\circ} \mathrm{C}$.

\section{Conclusions}

To summarize, we have performed polarized neutron diffraction experiment to characterize the magnetic nano-domain structures of the magnetic material exposed to the magnetic field at microwave frequencies. In the magnetite samples heated under magnetic field of microwave up to $1000^{\circ} \mathrm{C}$ and $1200^{\circ} \mathrm{C}$, magnetic Bragg peaks arising from crystalline magnetite of at least 20 nanometer in size were observed. In the latter sample the formation of non-magnetic substance, mostly hematite, was observed. These findings are consistent with the results of the magnetization measurement and X-ray diffraction. 